\title{
Diamante dopado com boro obtido pela técnica HFCVD para detecção eletroanalítica de metais
}

\section{Boron doped diamond electrode obtained by HFCVD technique for metals electroanalytical detection}

Neila A. Braga¹, Lidiane M. M. Ferreira¹, Maurício R. Baldan², Neidenêi G. Ferreira²

\section{RESUMO}

Neste trabalho, a técnica da Deposição Química a partir da Fase Gasosa foi utilizada para a deposição de filmes de Diamante Dopado com Boro (DDB) sobre substrato de Titânio. O eletrodo DDB assim obtido foi caracterizado morfológica e estruturalmente por Microscopia Eletrônica de Varredura, Difratometria de Raios X e por Espectroscopia de Espalhamento Raman. As condições experimentais utilizadas neste trabalho levaram à produção de filmes de diamante policristalino dopados, caracterizados por boa aderência, com predominância de carbono $\mathrm{sp}^{3}$. Estes filmes DDB foram também caracterizados eletroquimicamente por voltametria cíclica, apresentando uma janela de potencial de aproximadamente $3 \mathrm{~V}$. Os parâmetros cinéticos frente ao par redox $\mathrm{Fe}^{2+} / \mathrm{Fe}^{3+}$ foram levantados confirmando a característica de quase-reversibilidade dos filmes DDB. Após a caracterização dos referidos filmes, estes foram testados para a detecção dos metais $\mathrm{Cu}^{2+}$, $\mathrm{Fe}^{2+}$ e $\mathrm{Zn}^{2+}$ em meio aquoso. Os resultados mostraram que o eletrodo DDB é um material promissor para aplicação na área ambiental como sensor eletroquímico para a detecção destas espécies metálicas em águas contaminadas.

Palavras-chave: HFCVD; DDB; Detecção de metal.

\begin{abstract}
In this paper, Chemical Vapor Deposition technique was used for the deposition of Boron-doped Diamond (BDD) films on titanium substrate. The BDD electrode thus obtained was morphologically and structurally characterized by Scanning Electron Microscopy, X-ray Diffractometry and Raman Scattering Spectroscopy. The experimental conditions used in this study led to the production of doped polycrystalline diamond films characterized by good adhesion, with predominance of $\mathrm{sp}^{3}$ carbon. These BDD films were also electrochemically characterized by Cyclic Voltammetry, presenting a $\sim 3 \mathrm{~V}$ potential window. The kinetic parameters for the redox pair $\mathrm{Fe}^{2+} / \mathrm{Fe}^{3+}$ were raised confirming the quasi-reversibility feature of these BDD films. After its characterization, BDD films have been tested for the detection of metals $\mathrm{Cu}^{2+}, \mathrm{Fe}^{2+}$ e $\mathrm{Zn}^{2+}$ in aqueous media. The results showed that the BDD electrode is a promising material for application in the environmental area as electrochemical sensor for the detection of metal species in contaminated waters.
\end{abstract}

Keywords: HFCVD; BDD; Metal detection.

\footnotetext{
1Universidade Federal do Amazonas - Instituto de Ciências Exatas - Departamento de Química - Manaus (AM) - Brasil ${ }^{2}$ Instituto Nacional de Pesquisas Espaciais - Departamento de Engenharia e Tecnologias Espaciais - Laboratório Associado de Materiais e Sensores São José dos Campos (SP) - Brasil

Autor correspondente: Neila A. Braga - UFAM - Av. General Rodrigo Octávio, 6200, Coroado I - CEP 69077-000 - Manaus (AM) - Brasil

E-mail: neilalmeida_braga@hotmail.com

Recebido: 30/03/2015 Aprovado: 25/06/2015
} 


\section{INTRODUÇÃO}

Eletrodos formados por filmes de diamante dopado com boro (DDB) têm sido alvo das atenções de muitos pesquisadores ao longo dos anos ${ }^{(1-7)}$ devido a vantagens que incluem alta resistência à corrosão; ampla faixa de potencial de trabalho para a maioria dos solventes e eletrólitos; alta inércia química e eletroquímica; baixa sensibilidade ao oxigênio dissolvido; correntes residuais ou de fundo pequenas e estáveis, o que resulta numa excelente razão sinal/ruído; atividade eletroquímica reprodutível e alta condutividade elétrica $^{(8)}$.

O diamante em seu estado natural é um dos melhores isolantes presentes na natureza, podendo, porém, ter sua condutividade sensivelmente melhorada pela adição de dopantes $(\mathrm{B}, \mathrm{N}, \mathrm{O}, \mathrm{S})$ na rede cristalina deste material. Assim, a resposta eletroquímica dos eletrodos DDB depende do nível de dopagem dos filmes ${ }^{(9)}$, o qual pode originar filmes com propriedades eletrônicas que vão desde isolantes em baixas dopagens, para semicondutor ou até mesmo comportamento semimetálico, em altos níveis de dopagem.

No que se refere à detecção de espécies eletroativas, não apenas o eletrodo, mas também a técnica eletroanalítica utilizada é importante para aquisição de uma boa relação sinal ruído. A voltametria de redissolução anódica (VRA) tem sido muito utilizada na determinação de metais potencialmente tóxicos, uma vez que vários deles podem ser depositados na superfície do eletrodo através de eletrólise de soluções de seus íons.

Esta técnica abrange uma variedade de procedimentos eletroquímicos que incluem uma etapa de pré-concentração seguida por uma etapa voltamétrica. A etapa de pré-concentração consiste de uma eletrodeposição com potencial constante e controlado da espécie eletroativa sobre um eletrodo estacionário. Esta etapa é seguida por uma etapa de repouso e uma de determinação, sendo que esta última consiste na redissolução de volta à solução da espécie anteriormente eletrodepositada ${ }^{(10)}$.

Os métodos de redissolução são de vital importância nas análises envolvendo traços porque a etapa de eletrodeposição concentra o analito e permite a determinação de quantidades bastante baixas com razoável exatidão. Dessa forma, analitos na faixa de $10^{-6}$ a $10^{-9} \mathrm{~mol} \mathrm{~L}^{-1}$ podem ser determinados pelo método de redissolução anódica que emprega procedimentos simples e rápidos ${ }^{(11)}$.

Alguns trabalhos já foram realizados utilizando a voltametria de redissolução anódica. Giacomino e colaboradores ${ }^{(12)}$ determinaram mercúrio em água utilizando eletrodo de ouro maciço. Os parâmetros voltamétricos foram otimizados, a melhor resposta eletroquímica foi encontrada em solução diluída de $\mathrm{HCl}$ como eletrólito suporte, $150 \mathrm{~Hz}$ de frequência, 0,03 V amplitude e potencial de deposição $0 \mathrm{~V}$. O limite de detecção obtido foi $0,40 \mu \mathrm{g} \mathrm{L} \mathrm{L}^{-1}$.

Determinações individuais e simultâneas de chumbo, cádmio e zinco foram realizadas por Armstrong e colaboradores ${ }^{(13)}$ utilizando voltametria de redissolução anódica utilizando eletrodo de bismuto. Os limites de detecção encontrados foram 93,54 e $396 \mathrm{ng} \mathrm{L}^{-1}$ para $\mathrm{Pb}$ (II), Cd (II) e Zn (II) respectivamente.
Ndlov e colaboradores ${ }^{(14)}$ analisaram $\mathrm{Cu}^{2+}$ em amostras de água fortificadas. A análise envolveu uma etapa de acumulação de $-500 \mathrm{mV}$ sob agitação, seguido por voltametria de redissolução anódica acoplada com onda quadrada. Após a otimização dos parâmetros eletroquímicos obtiveram limite de detecção de $94 \mu \mathrm{g} \mathrm{L}^{-1}$ e recuperação entre $99 \%$ e $101 \%$.

Wotter ${ }^{(15)}$ realizou a especiação química do ferro e determinou a concentração deste metal total e dissolvido em amostras de águas intersticiais no sedimento do saco do Mendanha (RS). Foi utilizada a voltametria adsortiva de redissolução catódica com o ligante 2,3-dihidroxinaftaleno e voltametria de onda quadrada. $\mathrm{O}$ limite de detecção encontrado foi de $0,11 \mathrm{nmol} \mathrm{L}^{-1}$.

Langeloth e colaboradores ${ }^{(16)}$ fizeram um estudo voltamétrico com redissolução anódica e onda quadrada para detecção de zinco em meio amoniacal tamponado, os autores realizaram experimentos com a finalidade de obter os melhores parâmetros experimentais obtendo limites de detecção 5 ppb.

É perceptível que a técnica VRA é imprescindível para análise de diversos analitos. No entanto, considerar apenas as características vantajosas desta técnica não é suficiente ao decidir realizar um experimento voltamétrico. O eletrodo de trabalho é um elemento de fundamental importância e indispensável em uma célula eletroquímica. Isto é traduzido pelo número expressivo de pesquisas desenvolvidas com o objetivo de se obter novos eletrodos de trabalho, bem como otimizar e funcionalizar as superfícies destes eletrodos ${ }^{(17-21)}$. Estes trabalhos demonstram o desenvolvimento de novos tipos de eletrodos de trabalho sólidos para serem usados em voltametria. Embora boa parte ainda estejam sendo pesquisados, estes apresentam um considerável potencial para ampliar o campo de utilização da técnica voltamétrica. Dentre estes eletrodos, o DDB é o menos aplicado na detecção de metais, o que mostra a necessidade de estudos que contribuam para o entendimento dos mecanismos e aplicação deste eletrodo, o que é objeto de estudo neste artigo.

\section{PARTE EXPERIMENTAL}

\section{Crescimento dos filmes DDB}

Os eletrodos $\mathrm{DDB}, 1,0 \times 1,0 \mathrm{~cm}$ foram produzidos no Laboratório de Materiais Carbonosos do Laboratório Associado de Materiais e Sensores do Instituto Nacional de Pesquisas Espaciais (LABEMAC/LAS/INPE). Os mesmos foram produzidos a partir de um reator de filamento quente mostrado na Fig. 1A. A técnica utilizada foi a da deposição química a partir da fase gasosa ativada por filamento quente (HFCVD, do inglês Hotfilament Chemical Vapor Deposition). Os filmes foram crescidos em placas de titânio, as quais foram preparadas para o crescimento através de limpeza empregando a técnica de ultrassom utilizando acetona como solvente de extração das impurezas. Após a limpeza a superfície do titânio foi preparada para o crescimento por semeadura com pó de diamante $(0,25 \mu \mathrm{m})$ suspenso em hexano. 
A Fig. 1A apresenta um esquema da parte interna do reator que foi utilizado neste trabalho para a deposição dos filmes DDB. A temperatura de deposição foi atingida com aplicação de corrente nos filamentos de $125 \mathrm{~mm}$ a partir de uma fonte DC SUPLITEC. A corrente aplicada nos filamentos variou 13 a $16 \mathrm{~A}$. Os substratos foram posicionados no porta substrato a uma distância de aproximadamente $6 \mathrm{~mm}$ do filamento e mantidas a uma temperatura de aproximadamente $650{ }^{\circ} \mathrm{C}$ durante $10 \mathrm{~h}$. A temperatura do substrato, no reator, foi medida com um termopar de cromel-alumel posicionado à altura da superfície do substrato.

A Fig. 1B mostra o esquema da linha dos gases, bem como do processo de dopagem. Uma linha adicional de hidrogênio (1) passa através de um borbulhador contendo óxido de boro dissolvido em metanol numa concentração de 30000 ppm B/C, o qual é arrastado numa concentração que depende da temperatura, pressão de hidrogênio no borbulhador e fluxo de hidrogênio ${ }^{(22)}$. A pressão do hidrogênio no borbulhador foi mantida em aproximadamente $99,99 \mathrm{kPa}$ e a temperatura do borbulhador foi mantida em $30{ }^{\circ} \mathrm{C}$. A temperatura do borbulhador é um dos parâmetros mais importantes, pois ela está diretamente ligada à pressão de vapor do metanol. Quando $\mathrm{B}_{2} \mathrm{O}_{3}$ é dissolvido em $\mathrm{CH}_{3} \mathrm{OH}$, trimetilborato $\left(\mathrm{CH}_{3} \mathrm{O}\right)_{3} \mathrm{~B}$ é produzido, sendo a substância contendo boro adicionada à fase gasosa de crescimento.

Foi utilizado um rotâmetro para o controle do fluxo da mistura gasosa que saia do borbulhador para o interior do reator. O rotâmetro foi mantido em $40 \mathrm{sccm}$, o que foi equivalente, pela sua calibração, a uma concentração da mistura gasosa de cerca de $26 \%$. Esta mistura gasosa foi misturada com mais hidrogênio e metano, formando a mistura de crescimento de diamante dopado, composta de $1 \%$ de metano, 26\% de $\left(\right.$ metanol $+\mathrm{H}_{2}$ ) e $90 \%$ de hidrogênio. A pressão total dos gases mantida no reator foi de aproximadamente $5,33 \mathrm{kPa}$. Nas condições apresentadas neste trabalho, o nível de dopagem obtido neste trabalho corresponde à concentração de boro de 1,5 x $10^{21}$ átomos.cm ${ }^{-3}$, obtidas por medidas eletroquímicas (curvas Mott-Schottky) em trabalhos anteriores ${ }^{(23)}$.

\section{Caracterizações Estrutural e Morfológica dos Filmes DDB}

As caracterizações morfológicas e estruturais dos filmes DDB foram realizadas utilizando-se as técnicas da Microscopia Eletrônica de Varredura (MEV), Espectroscopia de Espalhamento Raman e Difratometria de Raios X. Essas técnicas foram importantes para o estudo dos filmes DDB, pois pela utilização destas foi possível analisar as características morfológicas, bem como a qualidade e estrutura dos mesmos. Abaixo segue uma breve descrição das condições experimentais e dos equipamentos utilizados.

\section{Microscopia Eletrônica de Varredura (MEV)}

As análises MEV foram realizadas em um microscópio QUANTA 250 da FEI COMPANY. As imagens foram capturadas em toda a superfície do eletrodo, nas bordas para verificar possíveis delaminações e no centro visto que este local é o utilizado durante as análises. As capturas foram realizadas em diversos aumentos sendo eles 24X; 30X; 1.000X e 10.000X. Para tais capturas foram utilizados os seguintes parâmetros experimentais: tensão de aceleração de $20 \mathrm{kV}$, distância de trabalho de $10 \mathrm{~mm}$, abertura do spot de 10, corrente de feixe de $60 \mathrm{~mA}$.

\section{Espectroscopia de Espalhamento RAMAN}

O espectro de espalhamento Raman apresentado neste trabalho, foi obtido usando um equipamento MicroRaman Sistema 2000 da Renishaw. A energia de excitação do laser de $\mathrm{Ar}^{+}$foi de 2,41 eV, correspondente ao comprimento de onda de $514,5 \mathrm{~nm}$. A profundidade de penetração do laser foi da ordem de $5 \mu \mathrm{m}$. Essa técnica foi importante para a identificação das fases de carbono diamante e não diamante presentes nos filmes depositados, bem como para avaliar a qualidade destes filmes em relação ao crescimento de diamante cristalino.

\section{Difratometria de Raios X (DRX)}

Os difratogramas RDX deste trabalho foram obtidos usando um sistema Philips X'Pert MRD, contendo um gerador PW-1830 e um controlador de difratômetro PW-1840, com
A

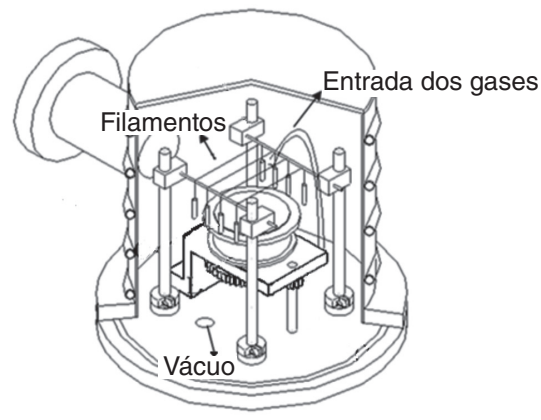

B

(2)

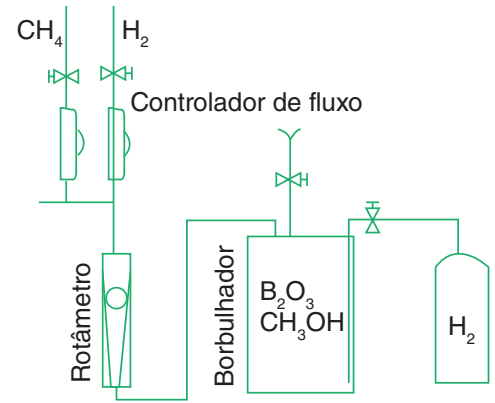

(1)

Figura 1: Desenho esquemático do reator de crescimento de filmes de diamante dopado com boro pela técnica HFCVD (A) Esquema da parte interna do reator-HCVD; (B) Sistema de dopagem. Fonte: Adaptada de Braga ${ }^{(22)}$. 
monocromador de grafite e radiação $\mathrm{CuKa}$,. Os espectros foram coletados com um passo de $0,02^{\circ}$ e 2,0 de integração. As análises DRX foram obtidas com valores de $2 \theta$ variando entre $30^{\circ}$ e $90^{\circ}$. A análise dos espectros obtidos foi feita tomando-se como referências, os padrões coletados na base de dados de difração do pó JCPDS - "Joint Committee on Powder Diffraction Standards"(24).

\section{Caracterizações Eletroquímicas do Eletrodo DDB}

Todas as vidrarias utilizadas nos experimentos eletroquímicos, como, célula eletroquímica, balões volumétricos, béqueres, foram previamente lavados com água destilada e deixados por 24 horas em uma solução de ácido nítrico $10 \%$ e então lavados com água ultra pura com resistividade de $18 \mathrm{Mohm} . \mathrm{cm}$ a $25^{\circ} \mathrm{C}$ tipo I (Milli-Q) antes do uso.

Os experimentos eletroquímicos realizados, neste trabalho, ocorreram em uma célula eletroquímica de vidro (Metrohm) $50 \mathrm{~mL}$. Tal célula foi constituída por três eletrodos cujo eletrodo de trabalho foi o DDB o auxiliar foi uma rede de platina e o de referência de $\mathrm{Ag} / \mathrm{AgCl}$. Tais eletrodos foram conectados a um potenciostato/galvanostato PGSTAT 302N - AUTOLAB com compliância de potencial de $+/-30 \mathrm{~V}$.

Todas as soluções utilizadas neste trabalho foram preparadas com reagentes de pureza analítica (PA). A deaeração das soluções (remoção de oxigênio) nas medidas voltamétricas foi realizada empregando-se o gás nitrogênio super seco 99,999\% (WHITE MARTINS) durante $180 \mathrm{~s}$.

Após cada experimento realizou-se a limpeza do eletrodo DDB. Esta limpeza ocorreu por um procedimento eletroquímico por voltametria cíclica onde foram feitas varreduras nos potenciais de $-2 \mathrm{~V}$ a $2 \mathrm{~V}$ por 30 minutos. Utilizou-se como solução de limpeza $\mathrm{H}_{2} \mathrm{SO}_{4} 0,5 \mathrm{~mol}$. L ${ }^{-1}$.

As caracterizações eletroquímicas dos filmes DDB foram realizadas conforme descrito abaixo:

\section{Janela de Potencial}

A observação da Janela de Potencial do eletrodo DDB foi realizada em célula eletroquímica cujos eletrodos estavam imersos em solução de $\mathrm{KCl}, 1 \mathrm{~mol} . \mathrm{L}^{-1}$, sem agitação. As voltametrias cíclicas foram realizadas em varreduras de potencial no intervalo de -3 a $3 \mathrm{~V}$ com uma velocidade de varredura igual a $10 \mathrm{mV} / \mathrm{s}$.

\section{Reversibilidade}

As medidas eletroquímicas necessárias ao estudo da reversibilidade do eletrodo DDB foram realizadas em soluções de $\mathrm{K}_{4} \mathrm{Fe}(\mathrm{CN})_{6} 1 \mathrm{mmol} \mathrm{L}^{-1}$. Esta solução foi preparada pela dissolução do sal em solução de $\mathrm{KCl} 1 \mathrm{~mol} \cdot \mathrm{L}^{-1}$. Os ensaios de voltametria cíclica foram obtidos em varredura de potencial no intervalo de $-0,15 \mathrm{~V}$ a $0,75 \mathrm{~V}$, além disso, foram realizados ensaios nas velocidades de varredura encontrada no intervalo de 5 a $100 \mathrm{mV} / \mathrm{s}$.

\section{RESULTADOS E DISCUSSÃO Caracterizações Morfológica, Estrutural e Eletroquímica dos Filmes DDB}

\section{Caracterizações Morfológica e Estrutural dos Filmes DDB}

Os eletrodos DDB, utilizados para o desenvolvimento desta pesquisa foram produzidos em lotes de quatro amostras, sendo necessário, para esta pesquisa, um total de dois lotes. Ambos os lotes foram produzidos com 30000 ppm de concentração de dopante. As condições de crescimento já foram descritas na parte experimental.

A Fig. 2A apresenta a imagem obtida por MEV da superfície dos filmes DDB obtidos neste trabalho, nas condições experimentais supracitadas. Através das imagens MEV dos filmes DDB, podese inferir que os mesmos cresceram em toda área do substrato sem presença de fissuras ou delaminações. Este fato é relevante, pois um problema comum relacionado ao crescimento de diamante em substratos de titânio encontra-se na diferença entre os coeficientes de expansão térmica destes dois materiais, sendo para o titânio $0,86 \times 10^{-5}\left(1 /{ }^{\circ} \mathrm{C}\right)$ e para o diamante $0,71 \times 10^{-5}\left(1 /{ }^{\circ} \mathrm{C}\right)^{(9)}$. Esta diferença causa um mal encaixe dos parâmetros de rede dos dois materiais, gerando tensões na rede cristalina que em grandes extensões podem causar as rachaduras ou delaminações dos filmes. Outro problema referente ao crescimento de filmes DDB relaciona-se com a formação de fases intermediárias como hidreto e carbeto de titânio. Estes fatores, em conjunto, fragilizam as amostras e podem ser responsáveis pela formação das referidas rachaduras e delaminações nos filmes $\mathrm{DDB}^{(22,25)}$. Portanto, o crescimento de filmes de diamante sobre substratos de titânio com boa qualidade é um grande desafio, o qual foi vencido neste trabalho através do ajuste dos parâmetros experimentais.

Morfologicamente observa-se visualmente na Fig. 2A, que o filme DDB policristalino é predominantemente formado por grãos de diamante microcristalinos. Tais grãos aleatoriamente orientados são formados por cristais bem facetados com orientação cristalográfica preferencial (111), determinada por difração de raios X (Fig. 2B).

As características morfológicas descritas favorecem a rugosidade do filme o que é eletroquimicamente importante, pois como foi verificado por Pleskov e colaboradores ${ }^{(26)}$, eletrodos com superfícies mais rugosas são mais reversíveis. Além disso, o aumento da rugosidade implica na melhora da área eletroquímica do eletrodo que resulta na elevação da resposta em corrente do mesmo ${ }^{(27)}$. A estrutura cristalina dos filmes DDB foi investigada por difração de raios X (DRX). Foram coletados padrões de raios $\mathrm{X}$ totais, com os ângulos $2 \theta$ no intervalo de $30^{\circ}$ e $90^{\circ}$. Os diagratogramas foram analisados com a base de dados JCPDSPDF 03-0858, JCPDS-PDF 32-1383 e JCPDS-PDF 73-0472(28). Através do espectro DRX, mostrado na Figura 2B é possível observar os picos em $2 \theta$ igual a $44^{\circ}$ e $75,5^{\circ}$ correspondentes às difrações dos raios X realizadas pelos planos (111) e (220) do 
diamante, confirmando a presença destas fases no filme, bem como a cristalinidade deste material.

O difratograma também mostra a formação da fase TiC (220) (311) $(222)^{(8)}$. Esta fase TiC é oriunda da difusão do carbono presente na atmosfera reacional para a matriz de titânio. A presença das fases TiC, mostra que a microestrutura do filme DDB se formou a partir de uma camada predominantemente composta de TiC. Durante o processo HFCVD, os átomos de carbono reagem com a superfície do Ti para formar grãos de TiC. Entretanto, à medida que a camada de carbeto atinge uma espessura crítica, a continuação do crescimento da fase de TiC é limitada pela capacidade do titânio de se auto-difundir até o substrato através da camada de $\mathrm{TiC}^{(29)}$. A partir daí o crescimento da fase diamante é privilegiada.

A

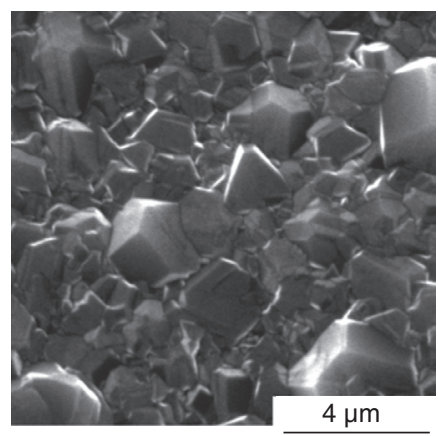

B

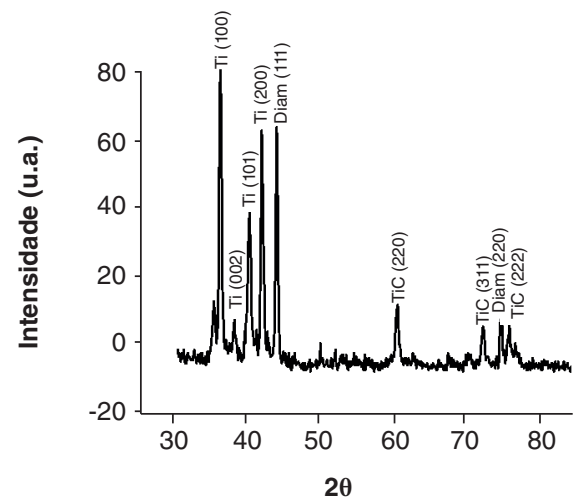

C

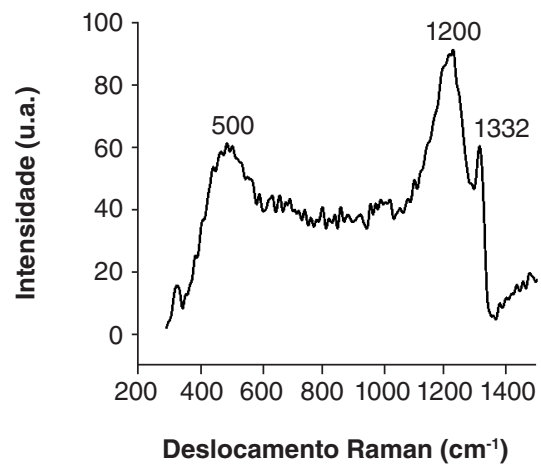

Figura 2: Caracterização morfológica e estrutural da superfície do filme DDB obtidos com solução de 30000 ppm de $\mathrm{B}_{2} \mathrm{O}_{3}$ em $\mathrm{CH}_{3} \mathrm{OH}$. (A) Imagem obtida por MEV (B) Difratograma de Raios X e (C) Espectro de Espalhamento Raman.
A estrutura das fases de carbono presentes nos filmes DDB foi analisada por espectroscopia de espalhamento Raman. A Fig. 2C apresenta o espectro vibracional obtido por esta técnica. Para todas as amostras obtidas, foi verificado um comportamento semelhante ao apresentado na Fig. 2B, ou seja, a presença da linha característica do diamante em $1332 \mathrm{~cm}^{-1}$ a qual corresponde à vibração do fônon de primeira ordem do diamante ${ }^{(30)}$.

É importante destacar o surgimento da banda em $1200 \mathrm{~cm}^{-1}$ que no caso de filmes DDB, é atribuída à desordem induzida na estrutura do diamante devido à incorporação de boro ${ }^{(31)}$, esta banda confirma a dopagem do filme de diamante. O nível de dopagem dos filmes utilizados neste trabalho correspondeu à concentração de boro de $1,5 \times 10^{21} \mathrm{~cm}^{-3}$ obtido por medidas eletroquímicas (curvas Mott-Schottky) em trabalhos anteriores ${ }^{(32)}$. Pode-se observar também o aparecimento de uma banda em torno de $500 \mathrm{~cm}^{-1}$. Esta banda pode ser atribuída aos modos de vibração de pares de boro, o que pode causar alguma distorção na rede do diamante ${ }^{(33)}$.

\section{Caracterizações Eletroquímicas dos Eletrodos DDB}

\section{Janela de Potencial do Eletrodo DDB}

A Janela de potencial, também denominada faixa de trabalho é um importante parâmetro a ser analisado uma vez que define a região que permite a detecção das espécies eletroativas ${ }^{(10)}$. Este ensaio foi realizado através de voltametria cíclica com varredura de potencial no intervalo de $-3 \mathrm{~V}$ a $3 \mathrm{~V}$ e velocidade de varredura $10 \mathrm{mV} / \mathrm{s}$. Conforme pode-se verificar pela análise do voltamograma apresentado na Fig. 3, a faixa de trabalho encontra-se aproximadamente no intervalo entre $-1,5 \mathrm{~V}$ a $1,5 \mathrm{~V}$, isso significa que o eletrodo DDB permite a detecção de substâncias que possuem potenciais bem mais positivos ou negativos sem que ocorra a interferência da eletrólise da água.

A partir do gráfico é possível verificar uma janela de potencial de aproximadamente $3 \mathrm{~V}$. Além disso, é possível observar as

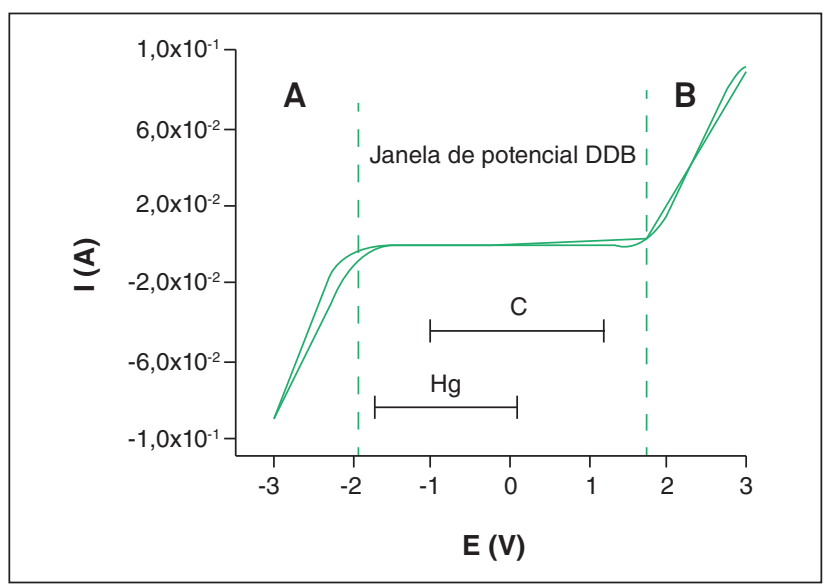

Figura 3: Voltamograma cíclico para o eletrodo DDB obtido em solução de $\mathrm{KCl} 1,0$ mol. $\mathrm{L}^{-1}$ e velocidade de varredura $10 \mathrm{mV} / \mathrm{s}$. Intervalos aproximados das Janelas de Potencial dos eletrodos de Carbono (C) e Mercúrio $(\mathrm{Hg})$ foram inseridos para título de comparação. 
regiões A e B onde ocorrem os processos de redução e oxidação da água respectivamente conforme as equações 1 e 2:

$2 \mathrm{H}_{2} \mathrm{O}+2 \mathrm{e}^{-} \leftrightarrow \mathrm{H}_{2}+2 \mathrm{OH}^{-}$

$\mathrm{H}_{2} \mathrm{O} \leftrightarrow 2 \mathrm{e}^{-}+1 / 2 \mathrm{O}_{2}+2 \mathrm{H}^{+}$

Os sinais em corrente gerados a partir das reações supracitadas podem mascarar o sinal em corrente gerado por qualquer analito que apresente potenciais de oxidação e redução nessas regiões, limitando, deste modo, as possibilidades de detecção do analito. Porém, o eletrodo DDB apresenta uma vantagem intrínseca que é o fato de os processos de oxidação e redução da água ocorrerem em altos potenciais ampliando assim a faixa de trabalho.

O eletrodo DDB obtido neste trabalho apresenta ampla faixa de trabalho quando comparado com outros dois eletrodos comercialmente disponíveis e muito utilizados em eletroanálise que são os eletrodos de carbono e de mercúrio. Na Figura 3, foram inseridas as Janelas de Potencial destes dois eletrodos para título de comparação. Desta forma, o eletrodo DDB apresenta o aspecto vantajoso de possibilitar a oxidação e redução de substâncias em altos potenciais. A exemplo de tais substâncias pode-se citar o $\mathrm{H}_{5} \mathrm{IO}_{6}$ cujo potencial de redução é $+1,60 \mathrm{~V}$ e o $\mathrm{PbO}_{2}+1,45 \mathrm{~V}^{(11)}$ tais substâncias possuem potenciais de redução inseridos na faixa de trabalho do DDB e fora das faixas dos demais eletrodos sendo impossível os processos de redução dessas substâncias nos eletrodos de carbono vítreo e mercúrio.

É importante destacar também que o eletrodo DDB possui baixa corrente de fundo que permite a detecção de analitos com irrelevante interferência de corrente capacitiva ${ }^{(34)}$. Para o eletrodo produzido neste trabalho, foi verificada uma faixa de ruído de $\left(10^{-5}\right.$ a $\left.10^{-6}\right)$ A de corrente. A medida da faixa de ruído foi realizada pela ampliação da região correspondente à janela de potencial mostrada na Figura 3. Esta medida foi realizada utilizando medidas de voltametria cíclica, mas o uso da técnica da voltametria de redissolução anódica melhora ainda mais este aspecto pelo fato de ser esta uma técnica de maior sensibilidade comprovada em inúmeros trabalhos na literatura quando comparada com a voltametria cíclica ${ }^{(10)}$.

\section{Área Ativa e Reversibilidade do $D D B$}

A Fig. 4 apresenta o conjunto dos voltamogramas cíclicos obtidos para o eletrodo DDB. Cada voltamograma cíclico foi obtido pela aplicação de uma diferença de potencial no intervalo de $-0,2 \mathrm{~V}$ a $0,7 \mathrm{~V}$. Foram coletados voltamogramas cíclicos em diferentes velocidades de varredura no intervalo de 5 a $100 \mathrm{mVs}^{-1}$. Os experimentos foram realizados em uma célula eletroquímica contendo solução de $\mathrm{K}_{4} \mathrm{Fe}(\mathrm{CN})_{6}$ 1,0 mmol. $\mathrm{L}^{-1}$ em $\mathrm{KCl}$ 1,0 mol.L $\mathrm{L}^{-1}$.

Para a realização dos ensaios o primeiro potencial aplicado foi de $-0,2 \mathrm{~V}$ x Ag/ $\mathrm{AgCl}$, onde foi possível observar a ocorrência de uma pequena corrente catódica que aproxima-se de zero a medida que a varredura prossegue. Essa corrente inicial surge da redução da água para formar hidrogênio e hidroxilas. Em 0,18 V e
0,34 V é possível verificar os picos em corrente para a redução e oxidação do ferro respectivamente, conforme a equação 3 :

$\mathrm{Fe}(\mathrm{CN})_{6}^{3-}+\mathrm{e}^{-} \leftrightarrow \mathrm{Fe}(\mathrm{CN})_{6}^{4-}$

A partir dos valores de corrente obtidos no voltamograma cíclico apresentado na Fig. 4 a área ativa do eletrodo foi calculada utilizando a equação 4 de Randles-Sevcik:

$\mathrm{Ip}=2,686 \times 10^{5} \mathrm{n}^{3 / 2} \mathrm{~A} \mathrm{C} \mathrm{D}^{1 / 2} \mathrm{v}^{1 / 2}$

Utilizando para o valor de coeficiente de difusão do ferrocianeto de potássio no diamante ${ }^{(35)}$ o valor de 7,26 $\times 10^{-6}$ encontrou-se a área ativa do eletrodo igual a $1,37 \mathrm{~cm}^{2}$. Desta forma, foi verificado um aumento de área do eletrodo em comparação com a área geométrica $\left(0,28 \mathrm{~cm}^{2}\right)$. Este resultado pode ser justificado a partir da análise das características morfológicas observadas na imagem obtida por MEV (Fig. 2A), tais como a predominância dos grãos de diamante microcristalinos, aleatoriamente orientados, formados por cristais bem facetados com preferencial orientação cristalográfica (111). Estas características favorecem a rugosidade do filme, o que resulta em uma área ativa maior do que a área geométrica do mesmo.

Em adição aos estudos já descritos, a cinética eletroquímica do filme DDB foi analisada. O objetivo foi verificar a resposta dos eletrodos DDB de acordo com os critérios de reversibilidade de reações de oxirredução. Com a finalidade de discutir a reversibilidade dos sistemas diamante $/ \mathrm{K}_{4} \mathrm{Fe}(\mathrm{CN})_{6}$ foram analisados os potenciais de pico anódico (Epa) e catódico (EPc), as intensidades das correntes de pico anódico (Ipa) e catódico (Ipc) em função da velocidade de varredura $(v)$.

A observação da Fig. 4 evidencia que um aumento na velocidade de varredura de potencial acarreta um aumento das intensidades das correntes de pico catódico (Ipc) e anódico (Ipa). Verifica-se que há um deslocamento do pico anódico para potenciais mais positivos e do pico catódico para o sentido de potenciais menores, o mesmo comportamento para o DDB também foi observado por Braga ${ }^{(22)}$.

Utilizando os critérios de reversibilidade foram confirmadas para o filme DDB as características de quase-reversibilidade.

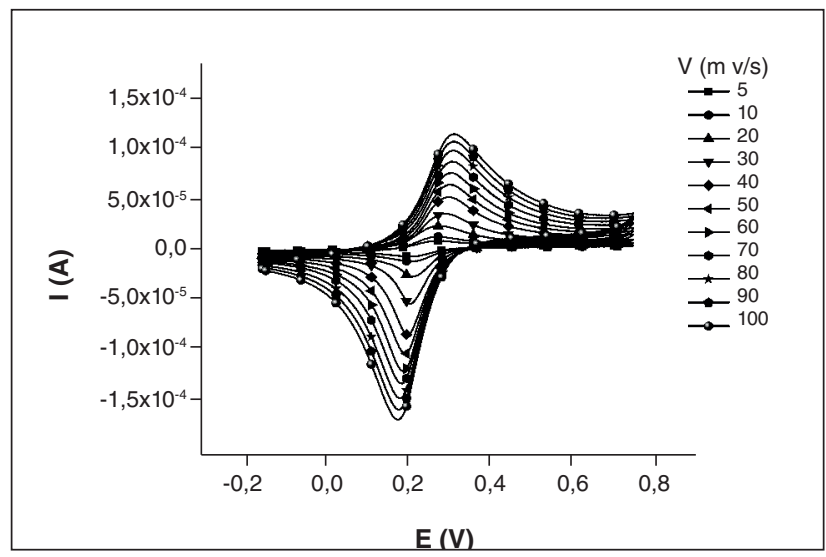

Figura 4: Voltamogramas cíclicos em $\mathrm{K}_{4} \mathrm{Fe}(\mathrm{CN})_{6} 1,0$ mmol.L-1 em KCl 1,0 mol. $\mathrm{L}^{-1}$ apresentados em diferentes velocidades de varredura. 
Esta confirmação deu-se pela observação de critérios como a separação entre os picos anódicos (Epa) e catódicos (Epc), que aumenta com o aumento da velocidade de varredura; ou o potencial de pico catódico, Epc, que varia caminhando no sentido de valores de potenciais mais negativos como uma função do aumento da velocidade de varredura e, ainda, a corrente de pico catódica que também aumenta com a raiz quadrada da velocidade de varredura ${ }^{(36)}$. Isto pode ser observado nos resultados apresentados nos gráficos das Figs. 5 e 6.

$\mathrm{O}$ gráfico apresentado na Fig. $5 \mathrm{~A}$ mostra a variação de $\Delta \mathrm{EP}$ com a velocidade de varredura, $(v)$ para os filme DDB em solução de Ferrocianeto $1 \mathrm{mmol} . \mathrm{L}^{-1} / \mathrm{KCl} 1 \mathrm{~mol} . \mathrm{L}^{-1}$. A sua observação revela que $\triangle \mathrm{EP}$ aumenta quando a velocidade de varredura é aumentada. A projeção da linha neste gráfico mostra que esta caminha para um valor próximo de $0,06 \mathrm{~V}$ em baixas velocidades de varredura. Isto mostra que, nesta condição, esse eletrodo aproxima-se da reversibilidade. Os valores de $\Delta \mathrm{EP}$ podem ser tomados como critério de reversibilidade, mesmo quando se desviam do valor teórico, desde que este desvio não seja muito grande $^{(26)}$. Logo, pode-se inferir da análise deste critério que o filme DDB apresenta cinética de quase-reversibilidade.

O comportamento da corrente de pico anódica (Ipa) em função da raiz quadrada da velocidade de varredura $\left(v^{1 / 2}\right)$ para o eletrodo em solução de ferrocianeto, é mostrado na Figura 5B. Nesta figura, pode se observar que há uma tendência de um aumento linear da corrente de pico anódica em função da raiz quadrada da velocidade de varredura. $\mathrm{O}$ fato do gráfico (Ipa $\mathrm{x} v^{1 / 2}$ ) apresentar

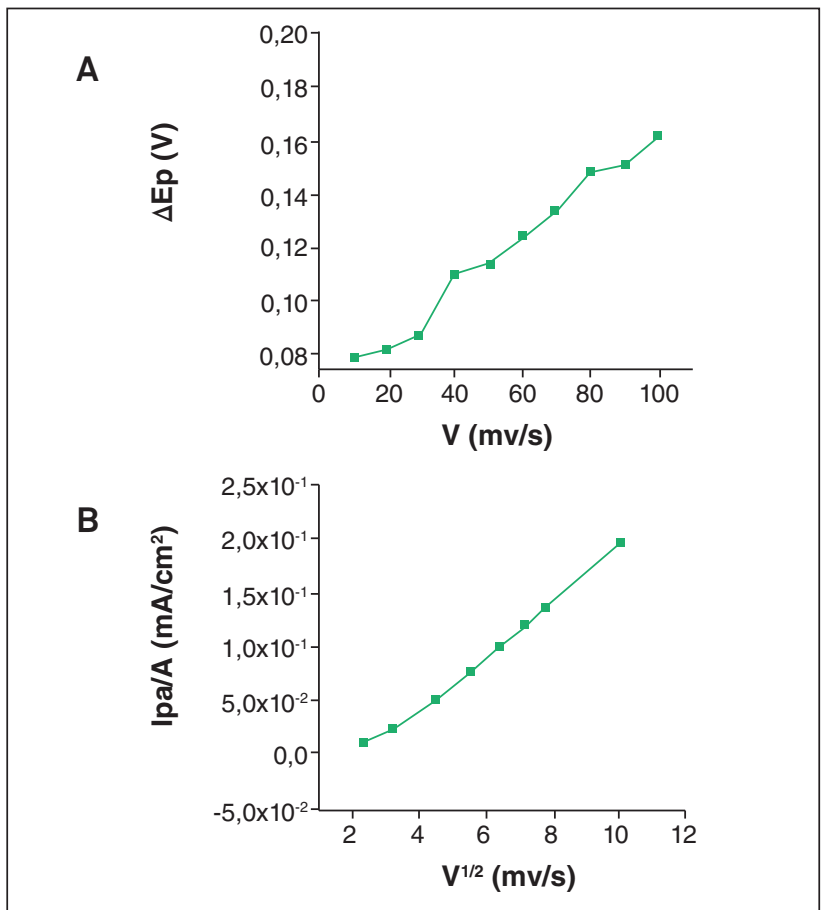

Figura 5: Parâmetros cinéticos do eletrodo DDB obtido em solução de $\mathrm{K}_{4} \mathrm{Fe}(\mathrm{CN})_{6} 1 \mathrm{mmol}^{-\mathrm{L}^{-1}} / \mathrm{KCl} 1 \mathrm{mmol}^{-L^{-1}}$ : (A) Relação entre a variação dos potenciais de Pico $(\triangle \mathrm{EP})$ e velocidade devarredura (v) e (B) Relação entre corrente de pico anódica (Ipa) e a raiz quadrada da velocidade de varredura $\left(v^{1 / 2}\right)$. um comportamento semelhante a uma reta confirma que a relação Ipa $/ \nu^{1 / 2}$ independe da velocidade de varredura, sendo este um critério de reversibilidade ${ }^{(36)}$. Este resultado sugere que o eletrodo deve apresentar cinética reversível apenas a baixas velocidades de varredura.

Apesar deste último critério apresentado apontar para a reversibilidade dos sistemas analisados, pode-se concluir que o conjunto de características apresentadas leva a concluir que o sistema DDB/ $\mathrm{K}_{4} \mathrm{Fe}(\mathrm{CN})_{6}$ é um sistema quase-reversível. A quase-reversibilidade das reações de oxi-redução investigadas com este eletrodo de diamante é resultante da baixa velocidade de transferência de elétrons sobre este eletrodo, insuficiente para manter o equilíbrio das espécies na interface eletrodo/solução.

\section{Efeito do Pré-Tratamento Superficial do Eletrodo DDB}

Conforme a literatura ${ }^{(8,23)}$ uma das importantes características do DDB é a não necessidade de tratamento superficial antes do uso. Porém, observou-se que em uma sucessão de ciclos não existia reprodutibilidade dos sinais em corrente de pico como mostra a Fig. 6A, onde são apresentados os voltamogramas cíclicos obtidos a partir da análise com $\mathrm{K}_{4} \mathrm{Fe}(\mathrm{CN})_{6} 1$ mmol.L $\mathrm{L}^{-1} \mathrm{em} \mathrm{KCl} \mathrm{1,0} \mathrm{mol.L} \mathrm{L}^{-1}$.

Um comportamento semelhante foi observado por Panizza, e colaboradores ao realizar cinco voltamogramas cíclicos em solução de 2-naftol 1,0x10-3 mol.L-1 em $\mathrm{H}_{2} \mathrm{SO}_{4} 1,0$ mol.L $\mathrm{L}^{-1}$, mas a atividade do eletrodo foi restabelecida após uma polarização anódica na solução do analito ${ }^{(37)}$.

Estudos realizados por Salazar-Banda e colaboradores demonstraram que ao aplicar um pré-tratamento ao eletrodo DDB

A

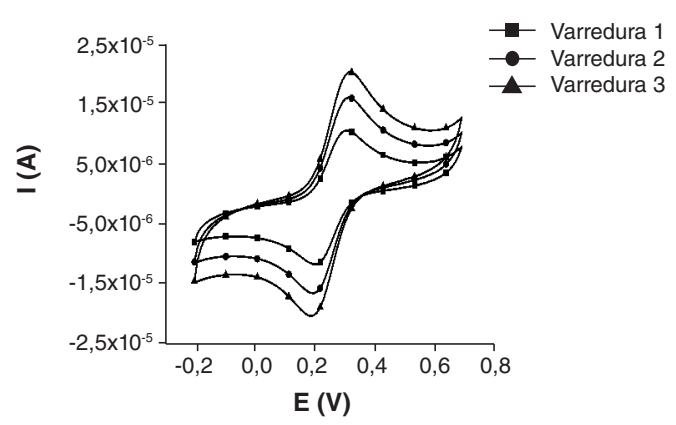

B

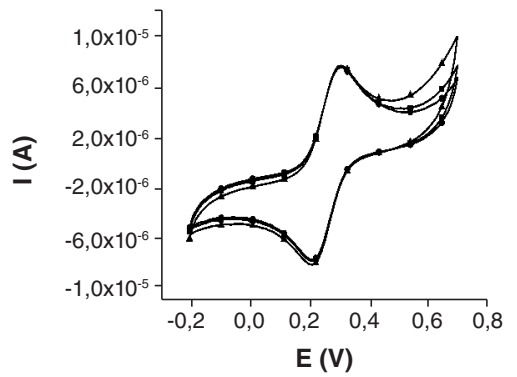

Figura 6: Voltamogramas cíclicos obtidos em solução de $\mathrm{K}_{4} \mathrm{Fe}(\mathrm{CN})$ $1,0 \mathrm{mmol} . \mathrm{L}^{-1} \mathrm{em} \mathrm{KCl} 1,0 \mathrm{~mol} . \mathrm{L}^{-1}$. Velocidade de varredura $30 \mathrm{mV} / \mathrm{s}$ : (A) Perfis voltamétricos anteriores ao pré-tratamento catódico e (B) Perfis voltamétricos após o tratamento catódico. 
é possível alcançar resultados reprodutíveis ${ }^{(38)}$. Tal tratamento consiste na polarização do eletrodo em $\mathrm{H}_{2} \mathrm{SO}_{4} 0,5$ mol.L-1 por 30 minutos em potencial de $+0,3 \mathrm{~V} / \mathrm{ECS}$, este procedimento é denominado polarização anódica. Após este processo é importante, também, realizar a polarização catódica em potencial de $-0,3 \mathrm{~V}$ nas mesmas condições anteriores.

A Figura 6B mostra os voltamogramas cíclicos obtidos após o pré- tratamento catódico do filme DDB. Este procedimento foi realizado conforme o procedimento adotado por Salazar-Banda ${ }^{(38)}$. Como demonstra a Fig. 6B após o tratamento catódico o eletrodo apresentou resultados reprodutíveis o que pode ser atribuído à renovação da superfície do eletrodo.

\section{Resposta Eletroquímica do Eletrodo DDB para Diferentes Eletrólitos Suporte}

Este estudo foi realizado em quatro tipos de eletrólitos suporte. Para tanto, preparou-se diferentes soluções de CuSO4 com os seguintes eletrólitos: $\mathrm{H}_{2} \mathrm{SO}_{4} ; \mathrm{CH}_{3} \mathrm{COOH} ; \mathrm{H}_{3} \mathrm{PO}_{4}$ e $\mathrm{KCl}$. Todas as soluções foram preparadas na concentração de $1,0 \times 10^{-3}$ mol.L $\mathrm{L}^{-1}$. Todas as soluções foram preparadas com reagentes de pureza analítica, e foram submetidas a análises de voltametrias de redissolução anódica acopladas à voltametria de onda quadrada. Para este procedimento utilizou-se a programação da VRA/VOQ com potencial de deposição $-0,2 \mathrm{~V}$, tempo de deposição 180 s, tempo de equilíbrio $60 \mathrm{~s}$, frequência $50 \mathrm{~Hz}$, amplitude $70 \mathrm{mV}$, potencial inicial $-0,2 \mathrm{~V}$ e potencial final $0,4 \mathrm{~V}$.

O eletrólito suporte é um importante parâmetro a ser considerado sobre a resposta eletroanalítica, pois tem influência na intensidade da corrente de pico ${ }^{(39)}$. A Fig.7 mostra a resposta em corrente para reação de oxidação do cobre em soluções de diferentes eletrólitos suporte.

A análise dos voltamogramas mostra que a definição do pico de oxidação do cobre em $\mathrm{CH}_{3} \mathrm{COOH}$ e $\mathrm{H}_{3} \mathrm{PO}_{4}$ foi comprometida devido à baixa intensidade da corrente de pico. $\mathrm{O}$ ácido sulfúrico proporcionou uma melhora na corrente, porém, o pico foi deslocado para potenciais negativos. Observa-se que o $\mathrm{KCl}$ favoreceu

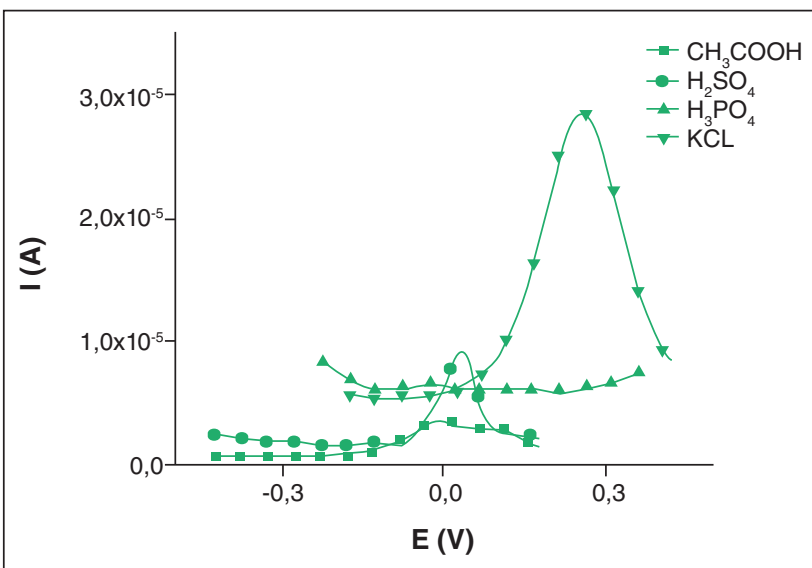

Figura 7: Resposta analítica VOQ/VRA para íons cobre na presença de diferentes eletrólitos suporte. Edep $=-0,1 \mathrm{~V}$, tempo de deposição $=60 \mathrm{~s}$, teq $=60 \mathrm{~s}, \mathrm{f}=50 \mathrm{~Hz}$ e a $=70 \mathrm{mv}$. a intensidade da corrente de pico, sendo este escolhido para dar continuidade aos estudos. Vale salientar que o mesmo comportamento foi observado para as análises de Zinco e Ferro.

\section{Comportamentos Voltamétrico do Cobre, Ferro e Zinco}

Com a finalidade de se verificar se o eletrodo DDB apresentava resposta eletroquímica aos íons metálicos Cobre $\left(\mathrm{Cu}^{2+}\right)$, Ferro $\left(\mathrm{Fe}^{2+}\right)$ e Zinco $\left(\mathrm{Zn}^{2+}\right)$, foram realizadas análises por voltametria cíclica destes três íons metálicos. Os voltamogramas foram obtidos em soluções de $\mathrm{CuSO}_{4}, \mathrm{ZnSO}_{4}$, e $\mathrm{FeSO}_{4} 1 \mathrm{mmol}$. $\mathrm{L}^{-1}$ em velocidades de varredura variando no intervalo de 5 a $100 \mathrm{mV} / \mathrm{s}$.

As análises das soluções dos metais por voltametria cíclica foram importantes para que fossem verificados os potenciais de oxidação-redução de cada analito. Os voltamogramas estão apresentados nas Figs. 8, 9 e 10. É importante destacar ainda, que os valores de potenciais de oxidação-redução, foram determinados considerando o voltamograma realizado em velocidade de $10 \mathrm{mV} / \mathrm{s}$ para cada metal.

A Fig. 8 mostra os voltamogramas cíclicos para os processos de oxidação-redução do cobre. A varredura partiu do potencial de $-0,6 \mathrm{~V}$ até $0,0 \mathrm{~V}$, posteriormente realizou-se a varredura no sentido inverso de $0,0 \mathrm{~V}$ até $-0,6 \mathrm{~V}$. Cada ciclo foi obtido em velocidades diferentes iniciando em $5 \mathrm{mV} / \mathrm{s}$ e finalizando em $100 \mathrm{mV} / \mathrm{s}$.

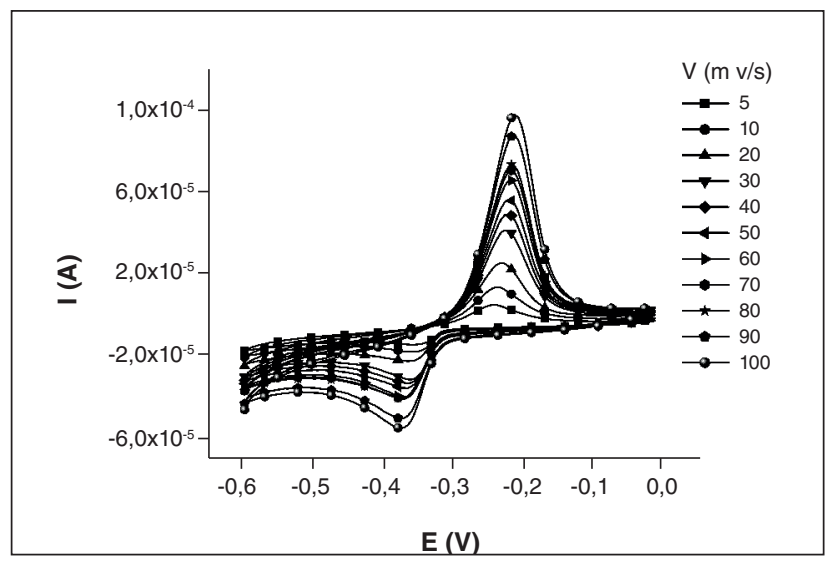

Figura 8: Voltamogramas cíclicos obtidos em diferentes velocidades de varredura em solução de $\mathrm{CuSO}_{4} 1 \mathrm{mmol}^{-L^{-1}} \mathrm{em} \mathrm{KCl} 1 \mathrm{~mol}^{\mathrm{L}} \mathrm{L}^{-1}$

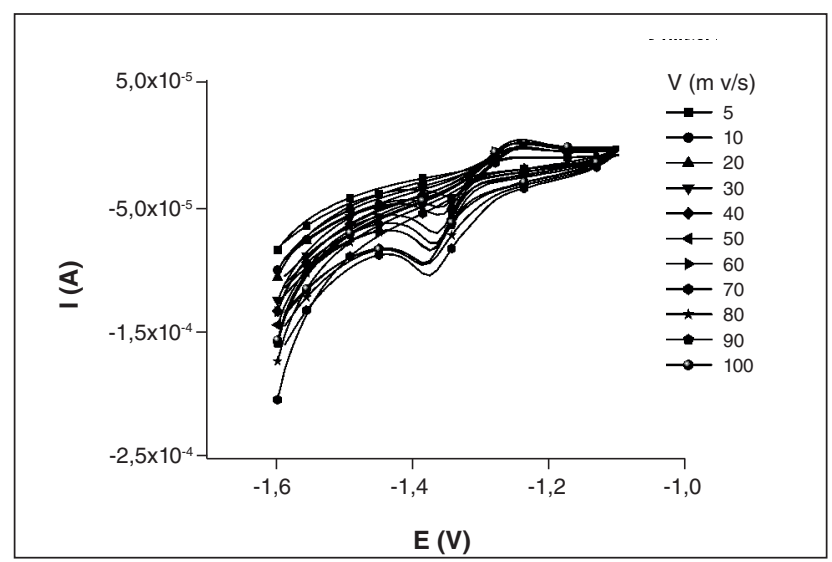

Figura 9: Voltamogramas cíclicos obtidos em diferentes velocidades de varredura em solução de $\mathrm{ZnSO}_{4} 1 \mathrm{mmol} . \mathrm{L}^{-1} \mathrm{em} \mathrm{KCl} 1$ mol. L-1. 


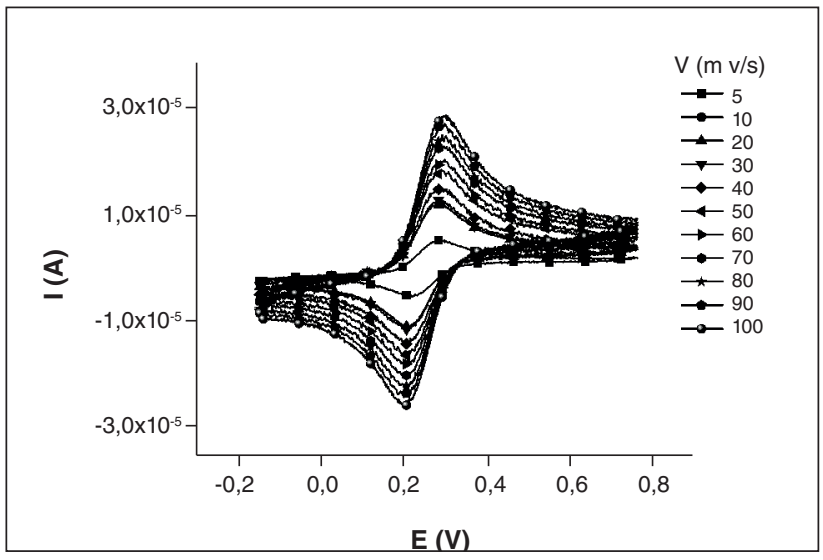

Figura 10: Voltamogramas cíclicos obtidos em diferentes velocidades de varredura em solução de $\mathrm{FeSO}_{4} 1 \mathrm{mmol}^{-\mathrm{L}^{-1}} \mathrm{em} \mathrm{KCl} 1 \mathrm{~mol}$. L-1.

A varredura no sentido anódico $(-0,6 \mathrm{~V}$ a $0,0 \mathrm{~V})$ revela o sinal em corrente associado à oxidação dos íons $\mathrm{Cu}^{0} \mathrm{a} \mathrm{Cu}^{2+}$. Este pico de corrente relaciona-se com o potencial de oxidação no qual o processo ocorre cujo valor é $-0,23 \mathrm{~V}$. Na varredura no sentido catódico $(0,0 \mathrm{~V}$ a $-0,6 \mathrm{~V})$ o pico de corrente catódico revelou um potencial catódico associado igual a $-0,35 \mathrm{~V}$.

A Fig. 9 apresenta os voltamogramas cíclicos para os processos redox do zinco. A varredura partiu do potencial de $-1,6 \mathrm{~V}$ até $-1,1 \mathrm{~V}$, posteriormente realizou-se a varredura no sentido inverso de $-1,1$ até $-1,6 \mathrm{~V}$. Cada ciclo foi obtido em velocidades diferentes iniciando em $5 \mathrm{mV} / \mathrm{s}$ e finalizando em $100 \mathrm{mV} / \mathrm{s}$.

A varredura no sentido anódico $(-1,6 \mathrm{~V}$ a $-1,1 \mathrm{~V})$ revela o sinal em corrente associado à oxidação dos íons $\mathrm{Zn}^{0} \mathrm{a} \mathrm{Zn}^{2+}$. Este pico de corrente esta relacionado com o potencial de oxidação do processo cujo valor é $-1,26 \mathrm{~V}$. Na varredura no sentido catódico $(-1,1 \mathrm{~V}$ a $-1,6 \mathrm{~V})$ o pico de corrente catódico e o potencial catódico associado igual a $-1,36 \mathrm{~V}$.

A Fig. 10 mostra os voltamogramas cíclicos para os processos redox do ferro. A varredura partiu do potencial de $-1,6 \mathrm{~V}$ até $-1,1 \mathrm{~V}$, posteriormente realizou-se a varredura no sentido inverso de $-1,1$ até $-1,6 \mathrm{~V}$. Cada ciclo foi obtido em velocidades diferentes iniciando em $5 \mathrm{mV} / \mathrm{s}$ e finalizando em $100 \mathrm{mV} / \mathrm{s}$.

A varredura no sentido anódico $(-0,2 \mathrm{~V}$ a $0,8 \mathrm{~V})$ revela o sinal em corrente associado à oxidação dos íons $\mathrm{Fe}^{0}$ a $\mathrm{Fe}^{2+}$. Este pico de corrente relaciona-se com o potencial em que este processo ocorre. Tal potencial é denominado potencial de oxidação cujo valor é $0,30 \mathrm{~V}$. Na varredura no sentido catódico $(0,8 \mathrm{~V}$ a $-0,2 \mathrm{~V})$ o pico de corrente catódico e o potencial catódico associado igual a $0,20 \mathrm{~V}$.

Como mencionado anteriormente, as análises por voltametria cíclica são importantes para o conhecimento dos potenciais redox dos metais em questão. Essas informações serão pontos de partida para os estudos de potenciais de deposição (parâmetro da VRA). Para os valores de potenciais de deposição, o equipamento utilizado nas análises, potenciostato/galvanostato PGSTAT - AUTOLAB realiza o arredondamento automático dos valores para apenas uma casa decimal, deste modo, os valores encontrados para os potencias redox do cobre, ferro e zinco serão utilizados conforme consta na Tabela 1.
Tabela 1: Potenciais de pico catódicos (Epc) e anódicos (Epa) para $\mathrm{Cu}, \mathrm{Fe}$ e $\mathrm{Zn}$.

\begin{tabular}{c|cc|}
\hline Analito & Epc (V) & Epa (V) \\
\hline $\mathrm{Cu}$ & $-0,3$ & $-0,2$ \\
$\mathrm{Fe}$ & 0,2 & 0,3 \\
$\mathrm{Zn}$ & $-1,4$ & $-1,3$ \\
\hline
\end{tabular}

\section{CONCLUSÕES}

Neste trabalho utilizou-se a técnica da Deposição Química na Fase Gasosa Assistida por Filamento Quente (HFCVD) para produzir eletrodos formados pela deposição de filmes de Diamante Dopado com Boro (DDB) sobre substrato de titânio. As condições experimentais estabelecidas no reator HFCVD geraram filmes que apresentaram uma superfície caracterizada pela presença de diamante policristalino. A análise por Espectroscopia Raman constatou a boa dopagem destes filmes com o elemento boro. Uma importante característica observada foi a boa aderência dos filmes no substrato de titânio com a predominância nestes de carbono do tipo $\mathrm{sp}^{3}$.

A caracterização eletroquímica dos filmes DDB mostrou que estes apresentaram uma ampla janela de potencial de aproximadamente $3 \mathrm{~V}$, o que torna o filme ideal para detecção de espécies eletroativas que não poderiam ser detectadas por eletrodos comercialmente disponíveis como os eletrodos de mercúrio e de pasta de carbono. Os parâmetros cinéticos frente ao par redox $\mathrm{Fe}^{2+} / \mathrm{Fe}^{3+}$ foram levantados confirmando a característica de quasereversibilidade dos filmes DDB e de tendência à reversibilidade em baixas velocidades de varredura.

A resposta eletroquímica destes eletrodos na detecção dos metais $\mathrm{Cu}^{2+}, \mathrm{Fe}^{2+}$ e $\mathrm{Zn}^{2+}$ em meio aquoso revelou os potenciais de oxidação e redução destas espécies na faixa de $-1,4 \mathrm{~V}$ a 0,3 V, ou seja, dentro da faixa de janela de potencial do eletrodo DDB $(-1,5 \mathrm{~V}$ a $1,5 \mathrm{~V}$ ). Isto evidencia que o eletrodo DDB é um material promissor para aplicação na área ambiental como sensor eletroquímico para a detecção voltamétrica destas espécies metálicas em águas contaminadas.

\section{AGRADECIMENTOS}

Os autores agradecem à CAPES, CNPq (processo $\mathrm{N}^{\circ}$ 458717/2014-2) e FAPEAM (processo $\mathrm{N}^{\circ}$ 06200542/2013) pelo fomento a esta pesquisa.

\section{REFERÊNCIAS}

1. VAZ, R.; MAY, P. W.; FOX, N.A.; HARWOOD, C. J.; CHATTERJEE, V.; SMITH, J. A.; HORSFIELD, C. J.; LAPINGTON, J. S.; OSBOURNE, $\mathrm{S}$. Measurement of the secondary electron emission from CVD diamond films using phosphor screen detectors. Journal of Instrumentation, v. 10, p. P03004-22, 2015 
2. CHATTERJEE, V.; HARNIMAN, R.; MAY, P.W.; BARHAI, P.K. Direct observation of electron emission from the grain boundaries of chemical vapour deposition diamond films by tunneling atomic force microscopy. Applied Physics Letters, v. 104, p. 171907-12, 2014.

3. ZANIN, H.; MAY, P. W.; SAITO, E.; MACHADO, J. P. B.; MARTINS, G.; TRAVA-AIROLDI, V. J.; CORAT, E. J. Effect of multi-walled carbon nanotubes incorporation on the structure, optical and electrochemical properties of diamond-like carbon thin films. Journal of Electrochemical Society, v. 161, p. H290-H295, 2014.

4. ANDRADE, H. D.; OTHMAN, M. Z.; O'DONNELL, K. M.; LAY, J. H.; MAY, P. W.; FOX, N. A.; MORIN, J.; RENAULT, O. Use of energyfiltered photoelectron emission microscopy and kelvin probe force microscopy to visualise work function changes on diamond thin films terminated with oxygen and lithium mono-layers for thermionic energy conversion. International Journal of Nanotechnology, v. 11, p. 796-807, 2014. DOI: 10.1504/IJNT.2014.063789.

5. HOPPER, A.; DUGAN, J.; GILL, A.; FOX, O.J.; MAY, P.W.; CLAEYSSENS, F. Amine functionalized nanodiamond promotes cellular adhesion, proliferation and neurite outgrowth. Biomedical Materials, v. 9, p. 045009-20, 2014

6. RIBEIRO, M. C. E.; COUTO, A. B.; MIGLIORINI, F. L,; FERREIRA, N. G.; BALDAN, M. R. Estudo da fotoeletrodeposição de partículas de cobre sobre filmes de diamante dopado com boro, Revista Brasileira de Aplicação de Vácuo, v. 32(1-2), p. 31-36, 2013. DOI: http://dx.doi.org/10.17563/rbav.v32i1-2.975.

7. PERALTA-HERNÁNDEZ, J. M.; MÉNDEZ-TOVAR, M.; GUERRASÁNCHEZ, R.; MARTÍNEZ-HUITLE, C. A.; NAVA, J. L. A brief review on environmental application of boron doped diamond electrodes as a new way for electrochemical incineration of synthetic dyes. International Journal of electrochemistry, v. 2012, Article ID 154316, 18 pages, 2012. DOI: http://dx.doi. org/10.1155/2012/154316

8. SUN, J.; LU, H.; LIN, H.; HUANG, W.; LI, H.; LU, J.; CUI, T. Boron doped diamond electrodes based on porous $\mathrm{Ti}$ substrates. Materials Letters, v. 83, p. 112-114, 2012. DOI:10.1016/j. matlet.2012.05.044.

9. MIGLIORINI, F. L. Produção e caracterização de eletrodos de diamante dopados com boro crescidos sobre titânio, aplicados na degradação de corante têxtil. Dissertação de mestrado (2011). Instituto Nacional de Pesquisas Epaciais, São José dos Campos (SP).

10. WANG, J. Analytical Electrochemistry. 3 ed. Wiley-VCH (Editores), p. $85-86,2006$

11. SKOOG, D. A.; HOLLER, F. J.; WEST, D. M.; CROUCH, S. R. Fundamentos de Química Analítica. 9 ed. Cengage Learning (Editores), p. 629-699, 2014.

12. GIACOMINO, A.; ABOLLINO, O.; MALANDRINO, M.; MENTASTI, E. Parameters affecting the determination of mercury by anodic stripping voltammetry using a gold electrode. Talanta, v. 75(1), p. 266-73, 2008

13. ARMSTRONG, K. C.; TATUM, C. E.; DANSBY-SPARKS, R. N.; CHAMBERS, J. Q.; XUE, Z.-L. Individual and simultaneous determination of lead, cadmium, and zinc by anodic stripping voltammetry at a bismuth bulk electrode. Talanta, v. 82(2), p. 67580. 2010. DOI: 10.1016/j.talanta.2010.05.031.

14. NDLOV U, T.; AROTIBA, O. A.; SAMPATH, S.; KRAUSE, R. W.; MAMBA, B. B. Electroanalysis of copper as a heavy metal pollutant in water using cobalt oxide modified exfoliated graphite electrode. Physics and Chemistry of the Earth, Parts A/B/C, v. 5052, p. 127-131, 2012. DOI:10.1016/j.pce.2012.08.007.

15. WOTTER, S. E. T. Desenvolvimento e validação de método voltamétrico para determinação de ferro e sua especiação química em água intersticial no sedimento da lagoa dos PatosRS. (2010). Dissertação de mestrado, Universidade Federal do Rio Grande, Rio Grande do Sul (RS).
16. LANGELOTH, M.; CHIKU, M.; EINAGA, Y. Anodic stripping voltammetry of zinc at borondoped diamond electrodes in ammonia buffer solution. Electrochimica Acta, v. 55(8), p. 2824 2828, 2010. DOI:10.1016/j.electacta.2009.12.097.

17. BARSAN, M. M.; GHICA, M. E.; BRETT, C. M. A. Electrochemical sensors and biosensors based on redox polymer/carbon nanotube modified electrodes: A review. Analytica Chimica Acta, v. 881, p. 1-23, 2015. doi:10.1016/j.aca.2015.02.059.

18. SUN, Y; ZHANG, W.; CHI, H.; LIU, Y.; HOU, C. L.; FANG, D.Recent development of graphene materials applied in polymer solar cell. Renewable and Sustainable Energy Reviews, v. p.973-980, 2015. doi:10.1016/j.rser.2014.11.040

19. SARANYA, K.; RAMEEZ, MD.; SUBRAMANIA, A. Developments in conducting polymer based counter electrodes for dye-sensitized solar cells - An overview. European Polymer Journal, v. 66, p. 207227, 2015. doi:10.1016/j.eurpolymj.2015.01.049.

20. KAKHKI, R. M. A review to recent developments in modification of carbon fiber electrodes. Arabian Journal of Chemistry, Article In Press. doi:10.1016/j.arabjc.2014.11.058

21. JIANG, S. P. Nanoscale and nano-structured electrodes of solid oxide fuel cells by infiltration: Advances and challenges. International Journal of Hydrogen Energy, v. 37(1), p. 449-470, 2012. doi:10.1016/j.ijhydene.2011.09.067.

22. BRAGA, N. A. Filmes de diamante-CVD sobre substrato de titanio puro poroso: uma proposta para aplicação como eletrodo. (2008). Tese de Doutorado, Instituto Nacional de Pesquisas Espaciais, São José dos Campos (SP).

23. FERREIRA, N. G.; SILVA, L. L. .; CORAT, E. J.; TRAVA-AIROLDI, V. J. Kinetics study of diamond electrodes at different levels of boron doping as quasi-reversible systems. Diamond and Related Materials, v. 11(8), p. 1523-1531, 2002. DOI:10.1016/S09259635(02)00060-2.

24. JOINT COMMITTEE ON POWDER DIFFRACTION STANDARDS (JCPDS). Base de dados do comitê internacional para padrões de difração do pó. [S.I]: JCPDS, 2002. 1 CD-ROM.

25. BRAGA, N. A.; CAIRO, C. A. A.; ALMEIDA, E. C.; M.R., B.; FERREIRA, N. G. From micro to nanocrystalline transition in the diamond formation on porous pure titanium. Diamond and Related Materials, v. 17(11), p. 1891-1896, 2008. DOI:10.1016/j. diamond.2008.04.002.

26. PLESKOV, Y.V.; EVSTEFEEVA, Y.E.; KROTOVA, M.D.; LIM, P.Y.; SHIH, H.C.; VARNIN, V.P.; TEREMETSKAYA, I.G.; VLASOV, I.I.; RALCHENKO, V.G. Synthetic diamons electrodes: The effectof surface microroughness on the electrochemical properties of CVD diamond thin films on titanium. Journal of Applied Electrochemistry, v. 35, n. 9, p. $857-864,2005$. DOI:10.1007/s10800-005-2572-0.

27. GOODALL, R.; MORTENSEN, A. 24 - Porous Metals. In: PHYSICAL METALLURGY. 5. ed. David E. Laughlin e Kazuhiro Hono (Editores), p. 2399-2595, 2014. DOI:10.1016/B978-0-444- 53770-6.00024-1.

28. JOINT COMMITTEE ON POWDER DIFFRACTION STANDARDS (JCPDS). Base de dados do comitê internacional para padrões de difração do pó. [S.I]: JCPDS, 2002. 1 CD-ROM.

29. SARIAN, S. Diffusion of Ti in TiC. Journal of Applied Physics, v. 40 (9), p. 3515-3520, 1969 DOI: 10.1063/1.1658229

30. KNIGHT, D. S.; WHITE, W. B. Characterization of diamond films by Raman spectroscopy. Journal of Materials Research, v. 4(2), p. 385-393, 1989. DOI: http://dx.doi.org/10.1557/JMR.1989.0385

31. YU, Z.; WANG, J.; WEI, Q.; MENG, L.; HAO, S.; LONG, F. Preparation, characterization and electrochemical properties of boron-doped diamond films on Nb substrates. Transactions of Nonferrous Metals Society of China, v. 23(5), p. 1334-1341, 2013. DOI:10.1016/S1003-6326(13)62601-1. 
32. SILVA, L. L. G. Eletrodos em diamante CVD para estudos eletroquímicos. (2001). Tese de Doutorado, Instituto Tecnológico de Aeronáutica, São José dos Campos (SP).

33. MAY, P. W.; LUDLOW, W. J.; HANNAWAY, M.; HEARD, P.J.; SMITH, J.A.; ROSSER, K.N. Raman and conductivity studies of borondoped microcrystalline diamond, facetted nanocrystalline diamond and cauliflower diamond films. Diamond and Related Materials, v. 17(2), p. 105-117, 2008. DOI:10.1016/j.diamond.2007.11.005

34. Zhou, Y; Zhi, J. The application of boron-doped diamond electrodes in amperometric biosensors. Talanta, v. 79 (5), p. 1189 1196, 2009. doi:10.1016/j.talanta.2009.05.026

35. CATTERALL, K.; MORRIS, K.; GLADMAN, C.; ZHAO, H.; PASCO, N.; JOHN, R. The use of microorganisms with broad range substrate utilization for the ferricyanide - mediated rapid determination of biochemical oxygen demand. Talanta, v. 55, p. 1187-1194, 2001. doi:10.1016/S0039-9140(01)00527-6.
36. GREEF R : PEAT, R : PETER, L. M : PLETCHER, D : ROBINSON, J. Instrumental methods in Electrochemistry. Woodhead Publishing (Editores), 2001.

37. PANIZZA, M.; MICHAUS, P. A.; CERISOLA, G.; COMNINELLIS C. Anodic oxidation of 2-naphthol at boron-doped diamond electrodes. Journal of Electroanalytical Chemistry, v. 507(1-2), p.206-214, 2001. DOI:10.1016/S0022-0728(01)00398-9.

38. SALAZAR-BANDA, G. R.; ANDRADE, L. S.; NASCENTE, P. A. P.; PIZANI, P. S.; ROCHA FILHO,R. C.; AVACA, L. A. On the changing electrochemical behaviour of boron-doped diamond surfaces with time after cathodic pre-treatments. Electrochimica Acta, v. 51(22) p. 4612-4619, 2006. DOI:10.1016/j.electacta.2005.12.039.

39. AGOSTINHO, S. M. L.; NETO, A. A.; VILLAMIL, R. F. V.; ARANHA $\mathrm{H}$. O eletrólito suporte e suas múltiplas funções em processos de eletrodo. Química Nova, v. 27(5), p. 813-817, 2004. http://dx.doi. org/10.1590/S0100-40422004000500022. 